\title{
Unveiled diversity: Amazonian Campinaranas harbor twice the number of bryophyte species recorded in the last century
}

\author{
Gabriela Ramos Cerqueira* (1), Adriel M. Sierra² (D), Anna Luiza Ilkiu-Borges ${ }^{3}$ (D), Sylvia Mota-de-Oliveira ${ }^{4}$ (1) \\ and Charles Eugene Zartman ${ }^{5}$ (D)
}

Received: September 21, 2020

Accepted: November 18, 2020

\begin{abstract}
Campinaranas are unique vegetation patches in the Amazonian biome, characterized by white-sand soils with relatively low nutrient content and subject to periodic flooding coupled with fluctuating groundwater levels. This study aimed to produce a synopsis of the bryophyte flora in Campinarana habitats by combining information from the literature with new collections made in the Uatumã Sustainable Development Reserve (Balbina, Central Amazonia), and areas of the middle Uaupés river (São Gabriel da Cachoeira, upper Rio Negro). One hundred and forty-three species were identified among mosses and liverworts, of which 68 are reported for the first time in Campinaranas. The species Frullania rio-janeirensis, Ceratolejeunea filaria, Diplasiolejeunea cobrensis and Bazzania diversicuspis are new records for the state of Amazonas. These results reveal high species richness for Campinaranas and indicate the need for continued study in this underexplored Amazonian habitat. This study emphasizes the importance of carrying out floristic inventories in poorly known environments and of further studies with different approaches, such as ecological, phytogeographic and genetic efforts.
\end{abstract}

Keywords: Amazon, bryophytes, Campinarana, rainforest, tropics

\section{Introduction}

Despite appearing homogeneous, Amazonia houses a wide variety of habitats favoring a vast diversity of life forms (Pires \& Prance 1985) which is increasingly subject to contemporary threats (Ellwanger et al. 2020).

Campinaranas are unique vegetation patches in the Amazonian biome, characterized by white-sand soils with relatively low nutrient content subject to periodic flooding coupled with fluctuating groundwater levels (Reichardt et al. 1975; Anderson 1981; Adeney et al. 2016).
White-sand ecosystems (including Campinaranas) covers approximately 5\% of the Amazon (Anderson 1981; Adeney et al. 2016). In Brazil, Campinaranas form vast areas on the upper Rio Negro and across the state of Roraima, but occur as fragmented patches or vegetation islands surrounded by Terra Firme forest in the Central and Lower Amazonia (Anderson 1981; Adeney et al. 2016).

Campinaranas, as opposed to Terra Firme forests, are composed by vegetation with a high degree of sclerophylly, low canopy and low productivity (Coomes \& Grubb 1996). Their species composition and distribution patterns have been the focus of several recent ecological studies,

\footnotetext{
1 Programa de Pós-Graduação em Botânica, Instituto Nacional de Pesquisas da Amazônia, 69067-375, Manaus, AM, Brazil

2 Département de Biologie, Université Laval, G1V 0A6, Quebec, Canada

3 Coordenação de Botânica, Museu Paraense Emílio Goeldi, 66077-530, Belém, PA, Brazil

4 Biodiversity Discovery Group, Naturalis Biodiversity Center, 2300 RA, Leiden, The Netherlands

5 Coordenação de Biodiversidade, Instituto Nacional de Pesquisas da Amazônia, 69067-375, Manaus, AM, Brazil
}

* Corresponding author: gabiibio@gmail.com 


\section{Unveiled diversity: Amazonian Campinaranas harbor twice \\ the number of bryophyte species recorded in the last century}

particularly analyzing the woody component such as trees and shrubs (García-Villacorta et al. 2016; Guevara et al. 2016), or vascular epiphytes, especially orchids (Braga 1978; Prance 1996; Klein \& Piedade 2019). These studies support the idea that Campinaranas harbor a typical assemblage of species related to the peculiarities of these environments. When compared to other vegetation types, they are less diverse but show a relatively high number of endemic, i.e. restricted, species. However, bryophytes (avascular plants) have not received the same attention as the other groups, being registered in these environments only in a few specific studies.

The first survey of bryophytes in Amazonian Campinarana was conducted by Lisboa (1976) in an area of Campina (a term used as a synonym for Campinarana) located at the $\mathrm{km} 62$ of the Manaus-Caracaraí road. This author considered the bryophytes, with 34 species, a wellrepresented group in comparison with the 45 species of woody plants reported by Anderson et al. (1975) for the same area. Earlier references of bryophytes in Amazonian Campinarana included only the genus Sphagnum (Ducke 1922; Ducke \& Black 1954; Egler 1960).

Griffin (1979) presented an annotated list of 147 bryophyte species collected in the greater Manaus region within a radius of $150 \mathrm{~km}$ of the capital of Amazonas State. Among the different vegetation types encompassed within his study area, Campinas harbored a bryophyte flora made up of 19 species with wide geographic distribution.

More recently, Sierra et al. (2018) presented a list of 150 species from different habitats in the Jaú National Park, Amazonas State, of which 34 occurred in Campinarana as well as other vegetation types, such as Terra Firme (upland) and Igapó forests (seasonally inundated blackwater forests).

Although commonly associated with preserved, humid and shaded places, bryophytes are also found in extreme environments such as deserts, pole regions and alpine elevations (Vittoz et al. 2010; Bramley-Alves et al. 2014; Smith \& Stark 2014). Bryophytes are avascular plants composed of species assemblages sensitive to environmental gradients. It is therefore expected that bryophyte assemblages in Campinaranas should also show specific patterns when compared to those of the relatively well-known Terra Firme forest. However, there is a deep gap in the knowledge that prevents such analysis. Among the 576 species of bryophytes listed for the Amazonia in the records of the repository Flora do Brasil 2020 (BFG 2018), only 15 are reported to occur in Campinaranas. After almost half a century from the first inventories, the number of bryophytes species from Amazonian Campinaranas in BFG (2018) is lower than the number of records provided by the literature, which highlights not only the need for more studies, but also the importance of a synthesis of the knowledge in this ecosystem type.

The fragmented nature of Campinaranas, the small area of their patches, and their susceptibility to anthropic disturbance place this vegetation type as one of the most threatened in the Amazonia (Silveira 2017). The lack of connectivity between these small patches hinders the recovery of locally extinct species (Álvarez-Alonso et al. 2013). The increasing threat in Amazonia forest calls attention to the need to protect this fragile ecosystem (Adeney et al. 2016).

Vascular plants depend on numerous factors for their establishment, but soil plays a predominant role. Bryophyte distribution, on the other hand, is mainly determined by niche and dispersal potential (Lönnel et al. 2012; Mota-de-Oliveira \& ter Steege 2015; Barbé et al. 2016; Garcia et al. 2020). Considering the richness recorded in the surrounding habitats, the low number of bryophyte species currently registered in Campinaranas is possibly a reflection of the lack of studies. In view of the bioindicator potential of these plants and the vulnerability of this type of ecosystem in Amazonia, assessing the bryophyte flora in these environments may contribute to the conservation of this biota.

The term Campinarana is associated with different phytophysiognomies called Campinarana Florestada (forested Campinarana), Campinarana Arborizada (wooded Campinarana), Campinarana Arbustiva (shrubby Campinarana) and Campinarana Gramíneo-lenhosa (grassywoody Campinarana or Campina) (IBGE 2012).

This study aimed to produce a synopsis of the bryophyte flora in Campinaranas, merging information from the literature and new collections in the Uatumã Sustainable Development Reserve (Balbina, Central Amazonia) and the vicinity of the Uaupés river (São Gabriel da Cachoeira, Upper Rio Negro).

\section{Materials and methods}

\section{Literature review}

The species list was based on previously published checklists, floristic inventories, ecological studies and taxonomic monographs in Amazonia, from which the information specifically related to species occurring in Campinaranas was filtered (Spruce 1884; Griffin 1975; Lisboa 1976; Reese 1993; Gradstein 1994; Gradstein \& Costa 2003; Sierra et al. 2018; Pereira 2019). Species specifically occurring in Campinaranas cited in BFG (2018) were also included.

\section{Sampling area}

Collections were carried out in two areas: the Uatumã Sustainable Development Reserve (USDR hereafter) and in the vicinity of the Uaupés river in São Gabriel da Cachoeira (SGC hereafter). Both areas are located in the state of Amazonas (Fig. 1). 
The USDR is located in the municipalities of Itapiranga and São Sebastião do Uatumã, Central Amazonia, comprising an area of $4,244 \mathrm{~km}^{2}$ along the Uatumã river basin. The reserve harbors four types of forests: Terra Firme, Várzea and Igapó, and Campinarana (Koury et al. 2012). Collections were made in areas of Campinarana Arbustiva, in which herbs and shrubs predominate distributed among low trees up to $7 \mathrm{~m}$ tall and a clean and sometimes open understory; Campinarana Arborizada, which is a non-forested formation but with some trees that reach $12 \mathrm{~m}$; and Campinarana Florestada with trees over $20 \mathrm{~m}$ high and an understory with the presence of palm trees (Fig. 2). The study area in SGC comprised trails along the Uaupés river, covering approximately $191.1 \mathrm{~km}$ of Campinarana Florestada.

\section{Bryophyte collections}

In the USDR, collections were performed in the vertical and horizontal gradient of the forest, covering a distance of approximately $2.18 \mathrm{~km}$, including all Campinaranas.

In the vicinity of the Uaupés river in SGC, collections were made along trails in an opportunistic manner, including only the understory and maximizing the number of species sampled. Although canopy samples were not included, bryophytes growing on recently fallen branches were collected.

In the two study areas, all available substrates were explored, namely, living tree trunks, logs, rocks, soil and leaves (Fig. 3). The collection followed the techniques described by Yano (1984) and the specimens were sun dried.

\section{Identification and classification}

The identification was based on specialized literature (Yano 1992; Ireland \& Buck 1994; He 1999; Ilkiu-Borges 2006; Gradstein et al. 2001; Buck 2003; Dauphin 2003; Gradstein \& Costa 2003; Ilkiu-Borges \& Lisboa 2004; Gradstein \& Ilkiu-Borges 2009, 2015; Bastos 2012; Pócs et al. 2014; Shi \& Zhu 2015; Bastos et al. 2016; Gradstein 2016; 2017; Bastos \& Zartman 2017; Lima et al. 2018; Oliveira-da-Silva \& Ilkiu-Borges 2018; Bastos \& Gradstein 2020) and/or on the analysis by specialists. The classification system adopted was the one of Crandall-Stotler et al. (2009) for Marchantiophyta and Goffinet et al. (2009) for Bryophyta, with updates by Carvalho-Silva et al. (2017) for Sematophyllaceae and Shi et al. (2015) for the genus Dibrachiella.

The specimens were classified according to the substrate from which they were collected: corticolous - on barks of

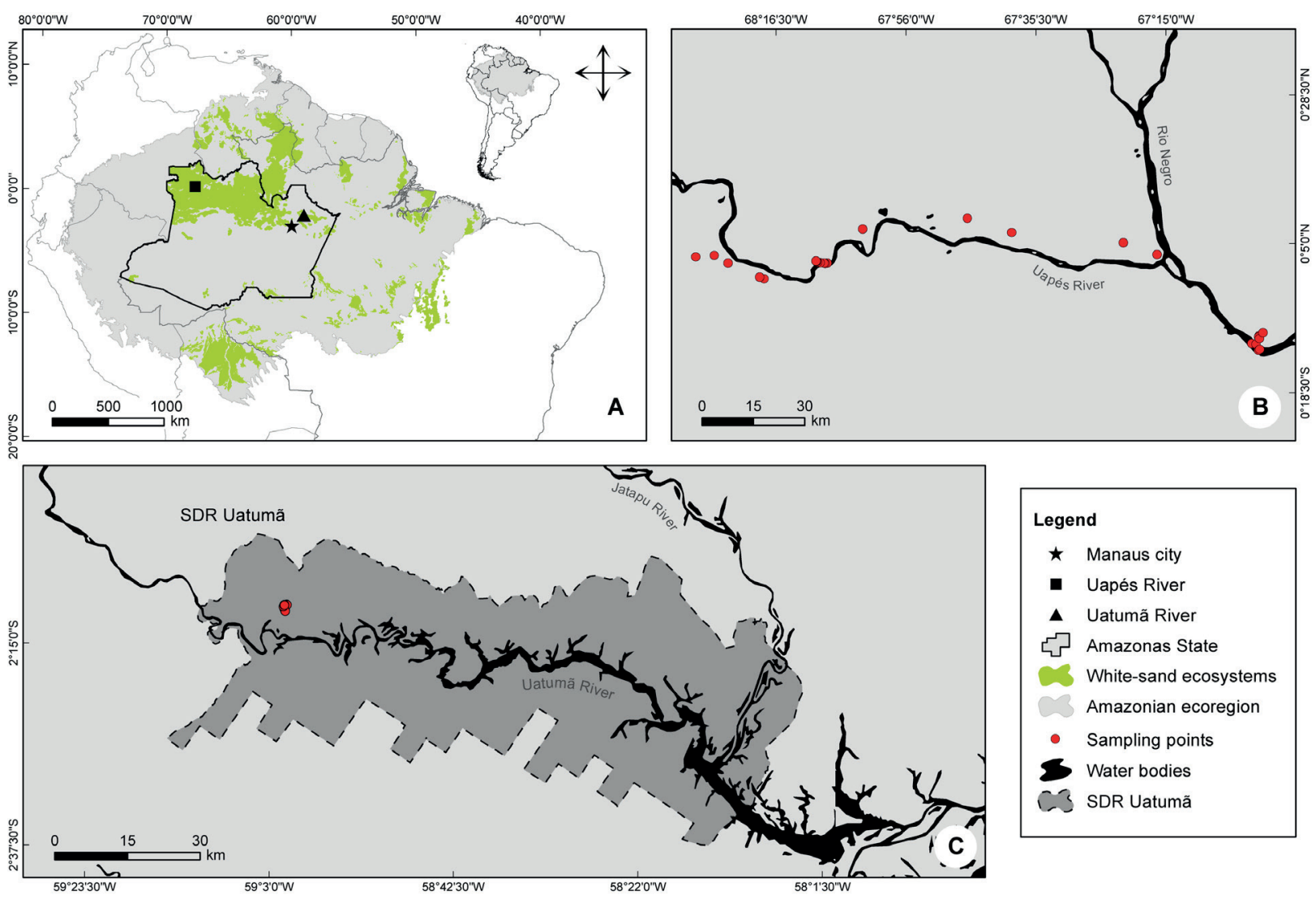

Figure 1. Map of the two study areas. A. Location of the two study areas in the Amazonas State and identification of Campinarana; B. Collection points along of the Uaupés river, municipality of São Gabriel da Cachoeira; C. Collection points in the Uatumã Sustainable Development Reserve, municipality of São Sebastião do Uatumã. 
Unveiled diversity: Amazonian Campinaranas harbor twice

the number of bryophyte species recorded in the last century
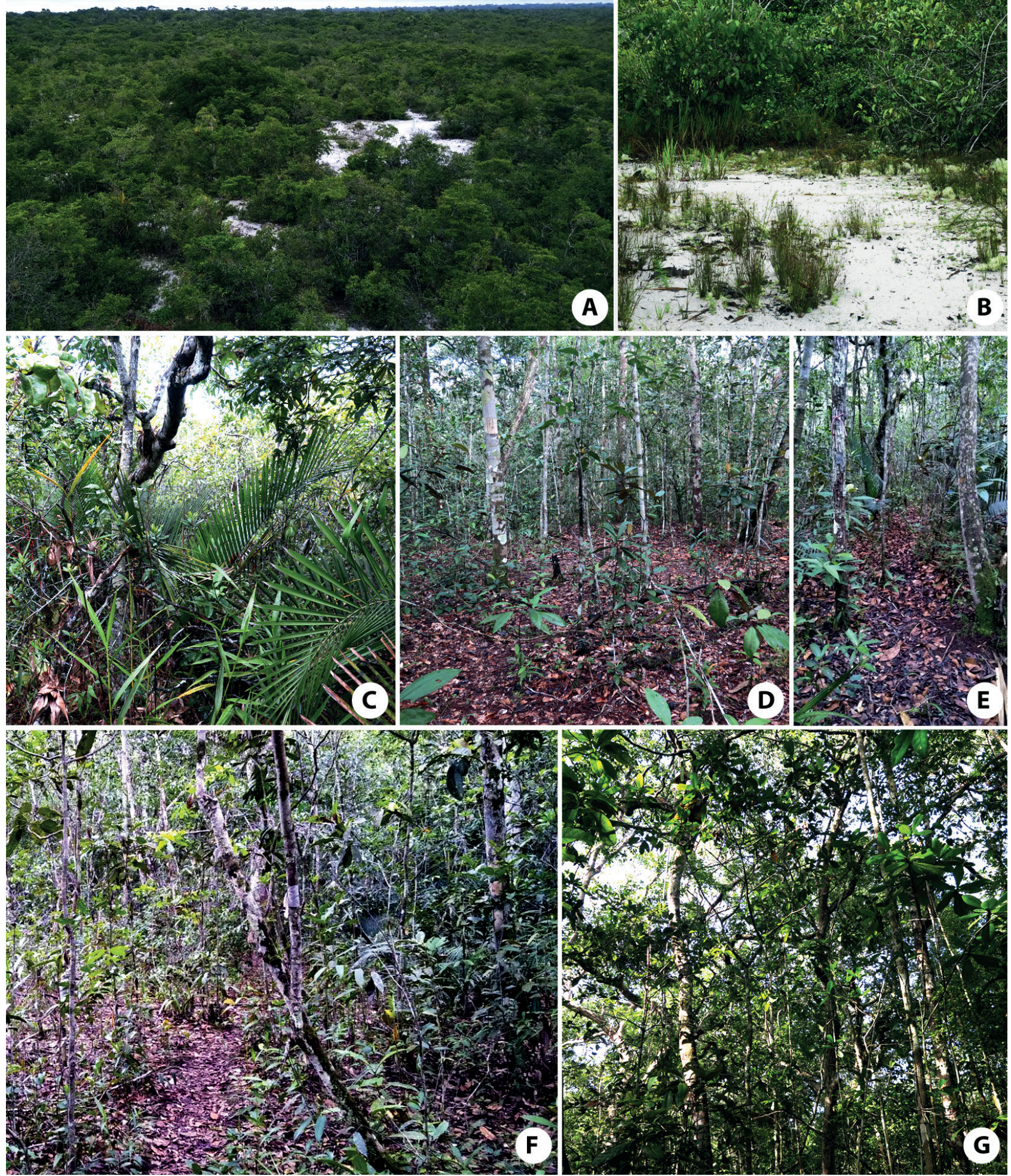

Figure 2. Field images of the collection areas in different physiognomies of Campinarana in the Amazonia. Campinarana arbustiva in aerial (A) and understory (B and $\mathbf{C})$ view. Campinarana arborizada seen from the understory $(\mathbf{D}$ and $\mathbf{E})$ and general $(\mathbf{F})$ view. Campinarana florestada in general view (G). Photos: Antônio Huxley Melo do Nascimento (A) and Charles E. Zartman (B-G). 

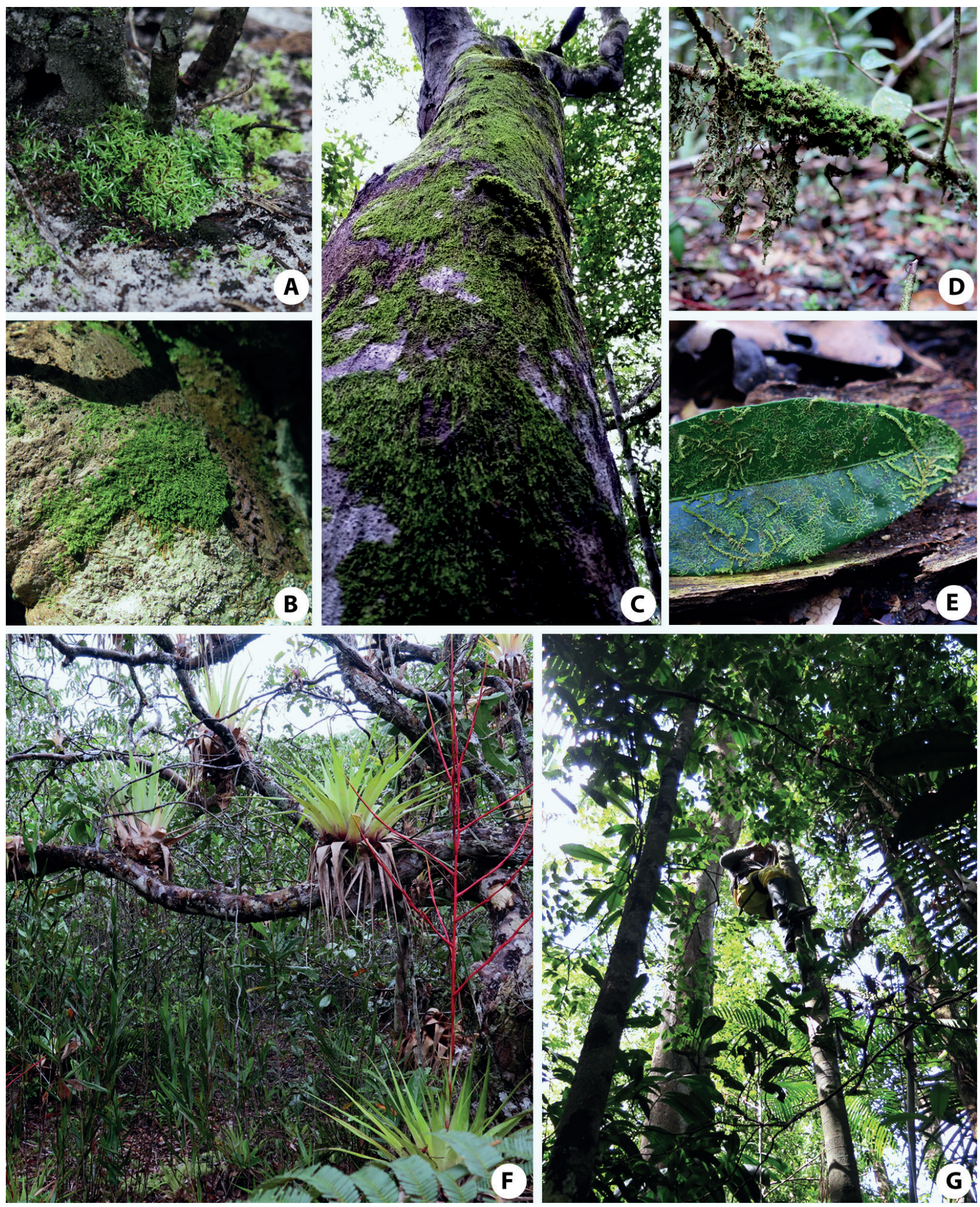

Figure 3. Substrates colonized by bryophytes in the study area. A. Soil in Campinarana arbustiva; B. Termite mound; C. Tree trunk; D. Tree branch; E. Living leaf; F. Understory of a Campinarana arbustiva; G. Tree climber collecting samples in the canopy in a Campinarana florestada. All photos by Charles E. Zartman. 
living trees; epixylous - on decaying wood; epiphyllous on leaves; saxicolous - on rocks; and terricolous - on soil (Robbins 1952). As for the world distribution patterns, the species were classified as Pantropical, Neotropical, Afroamerican, Amazonia, Amazonia-Guianas, Endemic in Brazil, Holartic regions, Madagascar, S Europe, SW Asia, Tropical South-American, and Trinidad (Fulford 1966; Reese 1993; Gradstein \& Costa 2003; Santos \& Costa 2010; Gradstein \& Uribe 2011; Santos et al. 2011; Gradstein 2013; Heirinchs et al. 2015; Carmo \& Peralta 2016; Pócs 2016; Gradstein 2017; BFG 2018; Pereira 2019).

Exsiccates will be deposited in the INPA herbarium from the Instituto Nacional de Pesquisas da Amazônia.

\section{Results and discussion}

Collections from the USDR, margins of the Uaupés river in SGC, and different Amazonian Campinaranas reported by Lisboa (1976), Griffin (1979), Sierra et al. (2018) and BFG (2018) resulted in a list of 143 species, of which $68 \%$ are liverworts (97 spp., 42 genera, 10 families) and $32 \%$ mosses (46 spp., 24 genera, 13 families) (Tab. 1).

A total of 116 species were identified in the 1273 specimens collected in Campinaranas of the USDR and SGC, and 68 of them are reported for the first time in this vegetation. Frullania rio-janeirensis, Ceratolejeunea filaria, Diplasiolejeunea cobrensis and Bazzania diversicuspis are new records for the state of Amazonas. These results reveal a high richness of species in Campinaranas and indicate the need for systematized sampling effort in this yet unexplored ecosystem.

Of the total of 143 species found in Campinaranas, 46 were recorded during the new collections and in the literature, whereas 20 are found in literature records only. Six species were solely reported by Lisboa (1976), seven by Griffin (1979), six by Sierra et al. (2018) and eight were exclusive to the list of BFG (2018). Some species were taxonomically updated, such as Cheilolejeunea clausa (cited as Eusmolejeunea suaveolens Spruce in Lisboa 1976), Frullania kunzei and Frullania intumescens (cited as Frullania neesi Lindb. and Frullania montagnei Gottsche in Griffin 1979, respectively).

In relation to the species registered in BFG (2018) for Amazonian Campinaranas (15 spp.), our list represents an addition of 128 species. In comparison with the current number of species registered by BFG (2018) for Terra Firme forest, the most investigated Amazonian vegetation type, Campinaranas harbor ca. $32 \%$ of the total richness of this environment. An early study on bryophytes in different Amazonian vegetation types in Colombia already indicated that species richness in white sand forests was not much lower than in other environments (Benavides et al. 2006). Using comparable sampling effort, the authors registered 32 species in the white-sand areas and 45 species in terra firme forests. According to Adeney et al. (2016), Campinaranas patches are usually overlooked because they are embedded in Terra Firme forests and therefore hardly detected in satellite images. Consequently, the number of species that occur in this vegetation type across the Amazon is also likely to be underestimated, especially of plant groups such as bryophytes.

Our results showed Lejeuneaceae as the most species rich family (64 spp.), followed by Calymperaceae (20 spp.), Lepidoziaceae (14 spp.), Sematophyllaceae (6 spp.) and Frullaniaceae (7 spp.) (Fig. 4). Lejeuneaceae is among the largest liverwort families and has a wide distribution, with a range of occurrence from rainforests to open formations, from lowlands to mountain ecosystems (Gradstein 1994; Gradstein et al. 2001; Carmo \& Peralta 2016; Oliveira-daSilva \& Ilkiu-Borges 2018). Lepidoziaceae is also a widely worldwide distributed family and in Brazil has been reported in the majority of the phytogeographic domains (Amazonia, Caatinga, Cerrado and Atlantic Forest) (Gradstein \& Costa 2003; BFG 2018). In turn, Frullaniaceae is a well-represented family in open vegetation types such as restinga, savanna, as well as in the understory and canopy of dense forest (Gradstein \& Costa 2003). These liverwort families were expected to occur in our study in view of the many types of Campinarana present in the USDR, varying from a grassy-woody Campinaranas with high luminosity and open understory to shaded forested Campinaranas with a species-rich understory and greater variety of substrates, including living leaves and decaying branches.

Among acrocarpous mosses, Calymperaceae was the most representative family with 20 species and Syrrhopodon Schwägr. was the genus with the largest number of species (10 spp.). The same result was recorded by PHM Sobreira (2018 unpubl. res.), who investigated the richness and composition of bryophyte and fern species in Campinarana and Terra Firme forest from two sites in Central Amazonia. Calymperaceae has high ecological and taxonomic importance in the Neotropics. More than half of its species reported to the Neotropics occur in the Brazilian Amazonia, where it is well represented in many of its ecosystems, including Campinaranas (Lisboa 1976; Gradstein et al. 2001; Pereira 2019). This family has a high rate of endemism and successfully colonizes different habitats, which is reflected on its high morphological variation (Reese 1993; Pereira 2019).

Corticolous bryophytes predominated in Amazonian Campinaranas. In this study, 116 were found on living tree trunks, 28 on decaying trunks, 18 on living leaves, 28 on rocks, and 26 on soil (Fig. 5). Some species were collected on more than one type of substrate, such as Calypogeia tenax, found growing on both decaying logs in SGC and rocks in the Jaú National Park (Sierra et al. 2018), and Ceratolejeunea coarina collected on both living trunks and living leaves in the USDR. The high colonization of living trunks by bryophytes can be explained by the great availability of 
Gabriela Ramos Cerqueira, Adriel M. Sierra, Anna Luiza Ilkiu-Borges,

Sylvia Mota-de-Oliveira and Charles Eugene Zartman

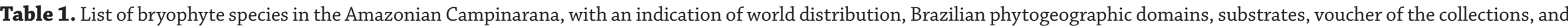

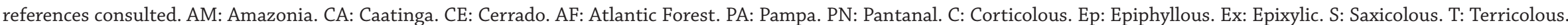
USDR: Uatumã Sustainable Development Reserve. SGC: São Gabriel da Cachoeira. ${ }^{*}$ New occurrence for the state of Amazonas. ${ }^{* *}$ First record in Campinarana in Central Amazonia.

\begin{tabular}{|c|c|c|c|c|c|}
\hline Taxa & World distribution & Phytogeographic domain & Substrate & $\begin{array}{l}\text { Voucher collections USDR } \\
\text { and SGC }\end{array}$ & $\begin{array}{l}\text { Reference (occurrence in } \\
\text { Campinarana) }\end{array}$ \\
\hline \multicolumn{6}{|l|}{ MARCHANTIOPHYTA (Liverworts) } \\
\hline \multicolumn{6}{|l|}{ Aneuraceae H.Klinggr. } \\
\hline Riccardia regnellii (Ångstr.) K.G.Hell & Tropical South-American & $\mathrm{AM}, \mathrm{CE}, \mathrm{AF}$ & Ex & Cerqueira 1201 & Sierra et al. (2018) \\
\hline \multicolumn{6}{|l|}{ Calypogeiaceae Arnell } \\
\hline Calypogeia tenax (Spruce) Steph. & Amazonian & AM & Ex, T & Sierra 5018 & Sierra et al. (2018) \\
\hline Mnioloma parallelogramum (Spruce) R.M.Schust. & Amazonian & $\mathrm{AM}, \mathrm{AF}$ & Ex, $S, T$ & Sierra 4851 & Sierra et al. (2018) \\
\hline${ }^{* *} M$. caespitosa (Spruce) R.M.Schust. & Tropical South-American & $\mathrm{AM}$ & C & Cerqueira 943 & - \\
\hline \multicolumn{6}{|l|}{ Cephaloziaceae Mig. } \\
\hline Odontoschisma brasiliense Steph. & Endemic to Brazil & $\mathrm{AM}, \mathrm{AF}$ & $C, E x, S$ & - & Griffin (1979) \\
\hline O. variabile (Lindenb. \& Gottsche) Trevis. & Afro-American (also in S Europe and SW Asia) & $\mathrm{AM}, \mathrm{CE}, \mathrm{AF}$ & C, Ex & Cerqueira 839,845 & $\begin{array}{c}\text { Griffin (1979) } \\
\text { Sierra et al. (2018) }\end{array}$ \\
\hline \multicolumn{6}{|l|}{ Frullaniaceae Lorch. } \\
\hline Frullania caulisequa (Nees) Mont. & Neotropical & $\mathrm{AM}, \mathrm{CA}, \mathrm{CE}, \mathrm{AF}, \mathrm{PA}$ & C & Cerqueira 929 & Sierra et al. (2018) \\
\hline${ }^{* *}$ F. exilis Taylor & Neotropical & $\mathrm{AM}, \mathrm{CE}, \mathrm{AF}$ & $C$ & Cerqueira 874, 899 & - \\
\hline F. gibbosa Nees & Neotropical & $\mathrm{AM}, \mathrm{CA}, \mathrm{CE}, \mathrm{AF}, \mathrm{PN}$ & $C, S$ & - & Griffin (1979) \\
\hline F. intumescens (Lehm. \& Lindenb.) Lehm. \& Lindenb. & Neotropical & $\mathrm{AM}, \mathrm{AF}$ & $C$ & - & Griffin (1979) \\
\hline F. kunzei (Lehm. \& Lindenb.) Lehm. \& Lindenb. & Neotropical & $\mathrm{AM}, \mathrm{CE}, \mathrm{AF}, \mathrm{PN}$ & C & Cerqueira 934 & Griffin (1979) \\
\hline F. nodulosa (Reinw. et al.) Nees & Pantropical & $\mathrm{AM}, \mathrm{AF}$ & C, Ex, T & Cerqueira 901 & $\begin{array}{l}\text { Lisboa (1976) } \\
\text { Griffin (1979) }\end{array}$ \\
\hline${ }^{*}$ F. rio-janeirensis (Raddi) Ångstr. & Pantropical & $\mathrm{AM}, \mathrm{CE}, \mathrm{AF}, \mathrm{PN}$ & C & Sierra 5081 & - \\
\hline \multicolumn{6}{|l|}{ Lejeuneaceae Cas. -Gil } \\
\hline Acrolejeunea torulosa (Lehm. \& Lindenb.) Schiffn. & Neotropical & $\mathrm{AM}, \mathrm{CE}, \mathrm{AF}, \mathrm{PN}$ & C & Cerqueira 841 Sierra 5036 & $\begin{array}{l}\text { Lisboa (1976) } \\
\text { Sierra et al. (2018) }\end{array}$ \\
\hline Archilejeunea badia (Spruce) Steph. & Amazonian & $\mathrm{AM}, \mathrm{AF}$ & C & Sierra 4830 & Sierra et al. (2018) \\
\hline A. crispistipula (Spruce) Steph. & Amazonian (and Guyana) & $\mathrm{AM}$ & c & Cerqueira 839 & $\begin{array}{l}\text { Sierra et al. (2018) } \\
\quad \text { BFG (2018) }\end{array}$ \\
\hline A. juliformis (Nees) Gradst. & Amazonian endemic & $\mathrm{AM}, \mathrm{AF}$ & C & Cerqueira 934 & Sierra et al. (2018) \\
\hline${ }^{* *}$ A. ludoviciana (Lehm.) P.Geissler \& Gradst. & Neotropical & AM & C & Sierra 4650 & - \\
\hline${ }^{* *}$ Caudalejeunea lehmanniana (Gottsche) A.Evans & Afro-American & $\mathrm{AM}, \mathrm{CE}, \mathrm{AF}, \mathrm{PA}$ & C & $\begin{array}{l}\text { Cerqueira } 981,994 \\
\quad \text { Sierra } 4877\end{array}$ & - \\
\hline Ceratolejeunea coarina (Gottsche) Schiffn. & Afro-American & $\mathrm{AM}, \mathrm{CE}, \mathrm{AF}$ & C, Ep & Cerqueira 847 & Sierra et al. (2018) \\
\hline${ }^{* *}$ C. confusa R.M.Schust. & Neotropical & $\mathrm{AM}, \mathrm{AF}$ & C & Cerqueira 855, 905 & \\
\hline C. cornuta (Lindenb.) Steph. & Afro-American & $\mathrm{AM}, \mathrm{AF}$ & C, Ex, Ep, S & Cerqueira 919, 937 & Lisboa (1976) \\
\hline C. cubensis (Mont.) Schiffn. & Neotropical & $\mathrm{AM}, \mathrm{AF}$ & C, Ex & Cerqueira 842 & Sierra et al. (2018) \\
\hline${ }^{* *}$ C. desciscens (Sande Lac.) Schiffn. & Amazonian (and Guyanas) & $\mathrm{AM}$ & C & Cerqueira 878 Sierra 5038 & - \\
\hline${ }^{*}$ C. filaria $($ Lehm.) Steph. & Neotropical & $\mathrm{AM}, \mathrm{AF}$ & C & Zartman 10192 & - \\
\hline C. rubiginosa Steph. & Neotropical & $\mathrm{AM}, \mathrm{AF}$ & C, Ep, Ex & - & Sierra et al. (2018) \\
\hline${ }^{* *}$ Cheilolejeunea acutangula (Nees) Grolle & Neotropical & $\mathrm{AM}, \mathrm{CE}, \mathrm{AF}$ & C, Ex & Cerqueira 860 & - \\
\hline
\end{tabular}


Table 1. Cont.

\begin{tabular}{|c|c|c|c|c|c|}
\hline Taxa & World distribution & Phytogeographic domain & Substrate & $\begin{array}{l}\text { Voucher collections USDR } \\
\text { and SGC }\end{array}$ & $\begin{array}{l}\text { Reference (occurrence in } \\
\text { Campinarana) }\end{array}$ \\
\hline${ }^{* *}$ C. adnata (Lehm.) Grolle & Neotropical & $\mathrm{AM}, \mathrm{AF}, \mathrm{PN}$ & C & Cerqueira 844 & - \\
\hline C. aneogyna (Spruce) A.Evans & Amazonia-Guianas & $\mathrm{AM}, \mathrm{AF}$ & C & Cerqueira 842 & Sierra et al. (2018) \\
\hline C. asperifolia (Spruce) Gradst. \& Ilk.-Borg. & Amazonian & $\mathrm{AM}$ & C & Cerqueira 915; Sierra 4665 & Sierra et al. (2018) \\
\hline C. clausa (Nees \& Mont.) R.M.Schust. & Neotropical & $\mathrm{AM}, \mathrm{CE}, \mathrm{AF}, \mathrm{PN}$ & C, Ex, S, T & - & Lisboa (1976) \\
\hline${ }^{* *}$ C. fragrantissima (Spruce) R.M.Schust. & South-American & $\mathrm{AM}$ & C & Sierra 4821 & - \\
\hline C. neblinensis Ilk.-Borg. \& Gradst. & Amazonian (and Bahia) & $\mathrm{AM}$ & C & Cerqueira 839; Sierra 4649 & Sierra et al. (2018) \\
\hline${ }^{* *}$ C. oncophylla (Ångstr.) Grolle \& M.E.Reiner & Neotropical & $\mathrm{AM}, \mathrm{AF}$ & C & Sierra 4827 & - \\
\hline${ }^{* *}$ C. papulosa Schiffn. & Amazonian & $\mathrm{AM}$ & C & Cerqueira 914; Sierra 4651 & - \\
\hline C. rigidula (Mont.) R.M.Schust. & Afro-American & $\mathrm{AM}, \mathrm{CA}, \mathrm{CE}, \mathrm{AF}, \mathrm{PN}$ & C, Ep & Cerqueira 919 & Sierra et al. (2018) \\
\hline${ }^{* *}$ C. trifaria (Reinw. et al.) Mizut. & Pantropical & $\mathrm{AM}, \mathrm{CE}, \mathrm{AF}, \mathrm{PN}$ & C & Zartman 10205 & - \\
\hline${ }^{* *}$ Cololejeunea appressa (A.Evans) Benedix & Pantropical & $\mathrm{AM}$ & Ep & Sierra 5027 & - \\
\hline${ }^{* *}$ C. camillii (Lehm.) A.Evans & Neotropical & $\mathrm{AM}, \mathrm{AF}$ & Ep & Sierra 4834 & - \\
\hline${ }^{* *}$ C. obliqua (Nees \& Mont.) Schiffn. & Pantropical & $\mathrm{AM}, \mathrm{AF}$ & C & Sierra 5033 & - \\
\hline${ }^{* *}$ C. surinamensis Tixier & South-American & $\mathrm{AM}, \mathrm{CE}, \mathrm{AF}$ & Ep & Sierra 5016 & - \\
\hline${ }^{* *}$ Colura tortifolia (Nees \& Mont.) Trevis. & Neotropical & $\mathrm{AM}, \mathrm{AF}$ & C, Ep & Sierra 5033 & \\
\hline${ }^{* *}$ Cyclolejeunea convexistipa (Lehm. \& Lindenb.) A.Evans & Neotropical & $\mathrm{AM}, \mathrm{AF}$ & C, Ep & Cerqueira 1045, 1046 & - \\
\hline${ }^{* *}$ C. luteola (Spruce) Grolle & Neotropical & $\mathrm{AM}, \mathrm{CE}, \mathrm{AF}$ & C, Ex, S & Sierra 4805 & - \\
\hline${ }^{* *}$ C. peruviana (Lehm. \& Lindenb.) A.Evans & Neotropical & $\mathrm{AM}, \mathrm{AF}$ & C, Ep & Zartman 10206 & - \\
\hline${ }^{* *}$ Dibrachiella parviflora (Nees) X.Q.Shi, R.L.Zhu \& Gradst. & Neotropical & $\mathrm{AM}, \mathrm{AF}$ & $\mathrm{C}$ & Cerqueira 847 & - \\
\hline${ }^{* *}$ Diplasiolejeunea brunnea Steph. & Neotropical & $\mathrm{AM}, \mathrm{CE}, \mathrm{AF}$ & C & $\begin{array}{c}\text { Cerqueira 846; Zartman } \\
10203\end{array}$ & - \\
\hline${ }^{* *}$ D. buckii Grolle & Amazonian & $\mathrm{AM}$ & C & Cerqueira 906, 909 & - \\
\hline${ }^{*}$ D. cobrensis Steph. & Neotropical & $\mathrm{AM}, \mathrm{CE}, \mathrm{AF}$ & C & Cerqueira 901, 903 & - \\
\hline Drepanolejeunea palmifolia (Nees) Schiffn. & Amazonian & $\mathrm{AM}, \mathrm{CE}, \mathrm{AF}, \mathrm{PN}$ & C, Ep & $\begin{array}{c}\text { Sierra } 4821 \\
\text { Zartman } 10198\end{array}$ & Sierra et al. (2018) \\
\hline${ }^{* *} D$. polyrhiza (Nees) Grolle \& R.-L. Zhu & Amazonian (and Guyanas) & $\mathrm{AM}$ & Ep & Sierra 5066 & - \\
\hline${ }^{* *}$ Lejeunea cerina (Lehm. \& Lindenb.) Lehm. \& Lindenb. & Neotropical & $\mathrm{AM}, \mathrm{AF}$ & C & Zartman 10217 & - \\
\hline${ }^{* *} L$. flava $($ Sw.) Nees & Pantropical & $\mathrm{AM}, \mathrm{CA}, \mathrm{CE}, \mathrm{AF}, \mathrm{PA}, \mathrm{PN}$ & C & Sierra 4877 & - \\
\hline${ }^{* *} L$. immersa Spruce & Amazonian (and Bahia) & $\mathrm{AM}, \mathrm{AF}$ & C & Sierra 4655 & - \\
\hline${ }^{* *}$ L. reflexistipula (Lehm. \& Lindenb.) Spruce & Neotropical & $\mathrm{AM}, \mathrm{CE}, \mathrm{AF}$ & C & Sierra 4836 & - \\
\hline${ }^{* *}$ Lepidolejeunea involuta (Gottsche) Grolle & Neotropical & $\mathrm{AM}, \mathrm{AF}$ & C, Ep, S, T & Sierra 4833 & - \\
\hline${ }^{* *}$ Leptolejeunea elliptica (Lehm. \& Lindenb.) Besch. & Neotropical & $\mathrm{AM}, \mathrm{CA}, \mathrm{CE}, \mathrm{AF}$ & C & Cerqueira 846 & - \\
\hline${ }^{* *} L$. moniliata Steph. & Amazonian (and Guyanas) & $\mathrm{AM}, \mathrm{AF}$ & Ep & Sierra 5066, 5070, 5071 & - \\
\hline${ }^{* *}$ Lopholejeunea subfusca (Nees) Schiffn. & Pantropical & $\mathrm{AM}, \mathrm{AF}, \mathrm{CA}, \mathrm{CE}, \mathrm{PN}$ & C & Cerqueira 938 & - \\
\hline L. eulopha (Taylor) Schiffn. & Pantropical & $\mathrm{AM}$ & C & Cerqueira 1200 & BFG (2018) \\
\hline Macrocolura sagittistipula (Spruce) R.M.Schust. & Neotropical & $\mathrm{AM}$ & C & Sierra 4820 & BFG (2018) \\
\hline${ }^{* *}$ Microlejeunea bullata (Taylor) Steph. & Neotropical & $\mathrm{AM}, \mathrm{CA}, \mathrm{CE}, \mathrm{AF}, \mathrm{PA}, \mathrm{PN}$ & C & Sierra 4820 & - \\
\hline${ }^{* *}$ Neurolejeunea seminervis (Spruce) Schiffn. & Neotropical & $\mathrm{AM}$ & C & Sierra 4845 & - \\
\hline
\end{tabular}


Gabriela Ramos Cerqueira, Adriel M. Sierra, Anna Luiza Ilkiu-Borges,

Sylvia Mota-de-Oliveira and Charles Eugene Zartman

Table 1. Cont.

\begin{tabular}{|c|c|c|c|c|c|}
\hline Taxa & World distribution & Phytogeographic domain & Substrate & $\begin{array}{l}\text { Voucher collections USDR } \\
\text { and SGC }\end{array}$ & $\begin{array}{l}\text { Reference (occurrence in } \\
\text { Campinarana) }\end{array}$ \\
\hline Odontolejeunea lunulata (F.Weber) Schiffn. & Pantropical & $\mathrm{AM}, \mathrm{CE}, \mathrm{AF}$ & C, Ep & Zartman 10206 & Lisboa (1976) \\
\hline Pictolejeunea sprucei Grolle & Amazonian & $\mathrm{AM}, \mathrm{AF}$ & $\mathrm{Ex}, \mathrm{S}$ & Sierra 4864 & Sierra et al. (2018) \\
\hline${ }^{* *}$ Prionolejeunea denticulata (F.Weber) Schiffn. & Neotropical & $\mathrm{AM}, \mathrm{AF}$ & C & Sierra 4827 & - \\
\hline${ }^{* *}$ Pycnolejeunea contigua (Nees) Grolle & Pantropical & $\mathrm{AM}, \mathrm{AF}$ & C & $\begin{array}{l}\text { Cerqueira } 876,904 \\
\quad \text { Sierra } 4657\end{array}$ & - \\
\hline P. macroloba (Nees \& Mont.) Schiffn. & Neotropical & $\mathrm{AM}, \mathrm{AF}$ & C, Ex & $\begin{array}{l}\text { Cerqueira 856, } 908 \\
\quad \text { Sierra } 4780\end{array}$ & $\begin{array}{l}\text { Lisboa (1976) } \\
\text { Sierra et al. (2018) }\end{array}$ \\
\hline${ }^{* *}$ P. remotistipula C.J.Bastos \& Zartman & Amazonian & $\mathrm{AM}$ & C & Cerqueira $860,867,910$ & - \\
\hline Schiffneriolejeunea amazonica Gradst. & Amazonian & AM & C & Cerqueira 918,939 & BFG (2018) \\
\hline${ }^{* *}$ Symbiezidium barbiflorum (Lindenb. \& Gottsche) A.Evans & Afro-american & $\mathrm{AM}, \mathrm{AF}$ & $\mathrm{C}$ & Cerqueira 1202, 1203 & - \\
\hline${ }^{* *}$ S. transversale (Sw.) Trevis. & Neotropical & $\mathrm{AM}, \mathrm{AF}$ & C, Ex & Sierra 5027 & - \\
\hline Thysananthus amazonicus (Spruce) Schiffn. & Neotropical & $\mathrm{AM}, \mathrm{CE}, \mathrm{AF}$ & C, Ex & Cerqueira 841, 912 & Lisboa (1976) \\
\hline **Verdoornianthus marsupiifolius (Spruce) Gradst. & Amazonian & $\mathrm{AM}$ & C & Cerqueira 874,877 & - \\
\hline${ }^{* *} V$. griffinii Gradst. & Amazonian (and French Guyana) & $\mathrm{AM}, \mathrm{AF}$ & C, Ep & Cerqueira 910, 916 & - \\
\hline $\begin{array}{c}\text { Vitalianthus aphanellus (Spruce) Bechteler, G.E.Lee, } \\
\text { Schäf.-Verw. \& Heinrichs }\end{array}$ & Tropical South American & $\mathrm{AM}, \mathrm{AF}$ & C & Sierra 5064 & Sierra et al. (2018) \\
\hline Xylolejeunea crenata (Nees \& Mont.) X.-L.He \& Grolle & Neotropical & $\mathrm{AM}, \mathrm{AF}$ & Ex, $S$ & $\begin{array}{l}\text { Cerqueira } 1047 \\
\text { Sierra } 4818\end{array}$ & $\begin{array}{l}\text { Sierra et al. (2018) } \\
\quad \text { BFG (2018) }\end{array}$ \\
\hline \multicolumn{6}{|l|}{ Lepidoziaceae Limpr. } \\
\hline${ }^{*}$ Bazzania diversicuspis Spruce & Tropical South American (and Trinidad) & $\mathrm{AM}$ & C & Cerqueira 854,857 & - \\
\hline B. hookeri (Lindenb.) Trevis. & Neotropical & $\mathrm{AM}, \mathrm{AF}$ & C & Cerqueira 842 & Sierra et al. (2018) \\
\hline B. longistipula (Lindenb.) Trevis. & Neotropical & $\mathrm{AM}, \mathrm{AF}$ & C & - & Sierra et al. (2018) \\
\hline B. pallidevirens (Steph.) Fulford & Tropical South American & $\mathrm{AM}, \mathrm{CE}, \mathrm{AF}$ & C, Ex & - & $\begin{array}{l}\text { Lisboa (1976) } \\
\text { Sierra et al. (2018) }\end{array}$ \\
\hline Micropterygium leiophyllum Spruce & Amazonian (+ Costa Rica) & $\mathrm{AM}, \mathrm{CE}, \mathrm{AF}$ & C, S, T & Sierra 5035 & Sierra et al. (2018) \\
\hline${ }^{* *}$ M. parvistipulum Spruce & Amazonian-Guianas & AM & $\mathrm{T}$ & Zartman 10194 & - \\
\hline${ }^{* *}$ M. pterygophyllum (Nees) Trevis. & Amazonian & $\mathrm{AM}, \mathrm{CE}, \mathrm{AF}$ & $C, E x, S, T$ & Zartman 10198 & - \\
\hline M. trachyphyllum Reimers & Neotropical & $\mathrm{AM}, \mathrm{CE}, \mathrm{AF}$ & C, Ex, S, T & Cerqueira 863 & $\begin{array}{l}\text { Lisboa (1976) } \\
\text { Sierra et al. (2018) }\end{array}$ \\
\hline Monodactylopsis monodactyla (Spruce) R.M.Schust. & Neotropical & $\mathrm{AM}, \mathrm{AF}$ & $\mathrm{T}$ & - & Sierra et al. (2018) \\
\hline${ }^{* *}$ Pteropsiella frondiformis Spruce & Amazonian & $\mathrm{AM}$ & $\mathrm{T}$ & Sierra 4814 & - \\
\hline P. metzgeriiformis R.M.Schust. & South-American & $\mathrm{AM}, \mathrm{AF}$ & $\mathrm{T}$ & Sierra 4781 & Lisboa (1976) \\
\hline Telaranea nematodes (Gottsche ex Austin) M.A.Howe & Afro-American & $\mathrm{AM}, \mathrm{CE}, \mathrm{AF}$ & $\mathrm{T}$ & - & Lisboa (1976) \\
\hline${ }^{* *}$ T. pecten (Spruce) J.J.Engel \& G.L.Merr. & Amazonian (and Guyanas and West Indies) & $\mathrm{AM}$ & $\mathrm{T}$ & Sierra 4870 & - \\
\hline Zoopsidella integrifolia (Spruce) R.M.Schust. & Neotropical & $\mathrm{AM}, \mathrm{CE}, \mathrm{AF}$ & $\mathrm{T}$ & Sierra 4825 & Sierra et al. (2018) \\
\hline \multicolumn{6}{|l|}{ Pallaviciniaceae Mig. } \\
\hline Symphyogyna brasiliensis (Nees) Nees \& Mont. & Afro-American & $\mathrm{AM}, \mathrm{CE}, \mathrm{AF}$ & $\mathrm{Ex}, \mathrm{S}, \mathrm{T}$ & - & Sierra et al. (2018) \\
\hline Plagiochilaceae (Joerg.) K. Müll. & & & & & \\
\hline
\end{tabular}


Unveiled diversity: Amazonian Campinaranas harbor twice

the number of bryophyte species recorded in the last century

Table 1. Cont.

\begin{tabular}{|c|c|c|c|c|c|}
\hline Taxa & World distribution & Phytogeographic domain & Substrate & $\begin{array}{l}\text { Voucher collections USDR } \\
\text { and SGC }\end{array}$ & $\begin{array}{l}\text { Reference (occurrence in } \\
\text { Campinarana) }\end{array}$ \\
\hline Plagiochila disticha (Lehm. \& Lindenb.) Lindenb. & Neotropical & $\mathrm{AM}, \mathrm{CA}, \mathrm{CE}, \mathrm{AF} \mathrm{PN}$ & C, S & Sierra 4838 & Sierra et al. (2018) \\
\hline P. montagnei Nees & Neotropical & $\mathrm{AM}, \mathrm{AF}$ & C, S & - & $\begin{array}{c}\text { Lisboa (1976) } \\
\text { Sierra et al. (2018) }\end{array}$ \\
\hline \multicolumn{6}{|l|}{ Radulaceae K. Müll. } \\
\hline${ }^{* *}$ Radula flaccida Lindenb. \& Gottsche & Afro-American & $\mathrm{AM}, \mathrm{AF}$ & C, Ep & Cerqueira 1204 & - \\
\hline${ }^{* *} R$. javanica Gottsche & Pantropical & $\mathrm{AM}, \mathrm{CE}, \mathrm{AF}, \mathrm{PN}$ & C & Zartman 10211 & - \\
\hline \multicolumn{6}{|l|}{ Ricciaceae L. } \\
\hline Riccia vitalii Jovet-Ast & Neotropical & AM, CA, CE, AF, PA, PN & $S, T$ & - & BFG (2018) \\
\hline \multicolumn{6}{|l|}{ BRYOPHYTA (Mosses) } \\
\hline \multicolumn{6}{|l|}{ Calymperaceae Kindb. } \\
\hline${ }^{* *}$ Calymperes bartramii W.D.Reese & South-American & $\mathrm{AM}$ & C & Zartman 10215 & - \\
\hline${ }^{* *}$ C. mitrafugax Florsch. & South-American & $\mathrm{AM}$ & C & Cerqueira 949 & - \\
\hline${ }^{* *}$ C. nicaraguense Renauld \& Cardot & Neotropical & $\mathrm{AM}, \mathrm{AF}$ & C & Cerqueira 1205 & - \\
\hline Octoblepharum albidum Hedw. & Pantropical & AM, CA, CE, AF, PA, PN & C & Cerqueira 982 & $\begin{array}{l}\text { Griffin (1979) } \\
\text { BFG (2018) }\end{array}$ \\
\hline O. ampullaceum Mitt. & South-American & AM & - & - & Griffin (1979) \\
\hline O. cocuiense Mitt. & Neotropical & $\mathrm{AM}, \mathrm{CE}, \mathrm{AF}$ & C, Ex & Cerqueira 839 Sierra 4785 & Griffin (1979) \\
\hline O. cylindricum Mont. & Neotropical & $\mathrm{AM}, \mathrm{CA}, \mathrm{CE}, \mathrm{AF}$ & $C, E x, T$ & Cerqueira 911 & $\begin{array}{l}\text { Lisboa (1976) } \\
\text { Griffin (1979) }\end{array}$ \\
\hline O. leucobryoides O.Yano & Endemic to Brazil & $\mathrm{AM}, \mathrm{AF}$ & C, T, S & Cerqueira 1206 & Sierra et al. (2018) \\
\hline O. pulvinatum (Dozy \& Molk.) Mitt. & Neotropical & $\mathrm{AM}, \mathrm{CA}, \mathrm{CE}, \mathrm{AF}, \mathrm{PN}$ & C, $\mathrm{T}$ & Cerqueira 1207 & $\begin{array}{l}\text { Lisboa (1976) } \\
\text { Griffin (1979) }\end{array}$ \\
\hline O. stramineum Mitt. & Neotropical & $\mathrm{AM}$ & C, $\mathrm{T}$ & Cerqueira 893 Sierra 4666 & $\begin{array}{l}\text { Lisboa (1976) } \\
\text { Griffin (1979) }\end{array}$ \\
\hline Syrrhopodon annotinus W.D.Reese \& D.G.Griffin & Amazonian & $\mathrm{AM}$ & C & Sierra 4662 & Sierra et al. (2018) \\
\hline${ }^{* *}$ S. cryptocarpus Dozy \& Molk. & Neotropical & $\mathrm{AM}, \mathrm{AF}$ & C & Cerqueira 846 & - \\
\hline S. fimbriatus Mitt. & South-American & AM & C & - & Lisboa (1976) \\
\hline **S. flexifolius Mitt. & Neotropical & $\mathrm{AM}$ & C & Cerqueira 1208 & - \\
\hline S. helicophyllus Mitt. & South-American & $\mathrm{AM}, \mathrm{AF}$ & C & Cerqueira 866 Sierra 4653 & Lisboa (1976) \\
\hline${ }^{* *}$ S. hornschuchii Mart. & South-American & $\mathrm{AM}, \mathrm{AF}$ & C & Sierra 4811 & - \\
\hline${ }^{* *}$ S. leprieurii Mont. & Neotropical & $\mathrm{AM}, \mathrm{AF}$ & C & Cerqueira 845 & - \\
\hline S. parasiticus (Brid.) Besch. & Pantropical & $\mathrm{AM}, \mathrm{CE}, \mathrm{AF}, \mathrm{PN}$ & C & - & Lisboa (1976) \\
\hline S. simmondsii Steere & Neotropical & $\mathrm{AM}$ & C & Cerqueira 839 Sierra 4864 & Sierra et al. (2018) \\
\hline S. xanthophyllus Mitt. & Neotropical & $\mathrm{AM}$ & C & Sierra 4861 & Sierra et al. (2018) \\
\hline \multicolumn{6}{|l|}{ Hypnaceae Schimp. } \\
\hline${ }^{* *}$ Phyllodon truncatulus (Müll. Hal.) W.R.Buck & Neotropical & $\mathrm{AM}, \mathrm{CE}, \mathrm{AF}$ & C & Sierra 4819 & - \\
\hline \multicolumn{6}{|l|}{ Hookeriaceae Schimp. } \\
\hline${ }^{* *}$ Crossomitrium Epyllum (Mitt.) Müll. Hal. & Neotropical & $\mathrm{AM}, \mathrm{AF}$ & Ep & Sierra 5080 & - \\
\hline
\end{tabular}


Gabriela Ramos Cerqueira, Adriel M. Sierra, Anna Luiza Ilkiu-Borges,

Sylvia Mota-de-Oliveira and Charles Eugene Zartman

Table 1. Cont.

\begin{tabular}{|c|c|c|c|c|c|}
\hline Taxa & World distribution & Phytogeographic domain & Substrate & $\begin{array}{c}\text { Voucher collections USDR } \\
\text { and SGC }\end{array}$ & $\begin{array}{l}\text { Reference (occurrence in } \\
\text { Campinarana) }\end{array}$ \\
\hline${ }^{* *}$ Hookeria acutifolia Hook. \& Grev. & Neotropical & $\mathrm{AM}, \mathrm{AF}$ & C & Sierra 5085 & - \\
\hline \multicolumn{6}{|l|}{ Leucobryaceae Schimp. } \\
\hline${ }^{* *}$ Campylopus savannarum (Müll. Hal.) Mitt. & Pantropical & $\mathrm{AM}, \mathrm{AF}, \mathrm{CA}, \mathrm{CE}, \mathrm{PN}$ & S & Cerqueira 1210 & - \\
\hline${ }^{* *}$ C. surinamensis Müll. Hal. & Neotropical & $\mathrm{AM}, \mathrm{AF}, \mathrm{CE}, \mathrm{PN}$ & S & Cerqueira 1209 & - \\
\hline Leucobryum albicans (Schwägr.) Lindb. & Neotropical & $\mathrm{AM}, \mathrm{CA}, \mathrm{CE}, \mathrm{AF}$ & $S, T$ & - & BFG (2018) \\
\hline L. crispum Müll. Hal. & Neotropical & $\mathrm{AM}, \mathrm{CE}, \mathrm{AF}$ & $S, T$ & - & Griffin (1979) \\
\hline L. martianum (Hornsch.) Müll. Hal. & Neotropical & $\mathrm{AM}, \mathrm{CA}, \mathrm{CE}, \mathrm{AF}, \mathrm{PN}$ & $C, E x, T$ & Cerqueira 839 & $\begin{array}{c}\text { Griffin (1979) } \\
\text { Sierra et al. (2018) }\end{array}$ \\
\hline Ochrobryum gardneri (Müll. Hal.) Lindb. & Afro-American & $\mathrm{AM}, \mathrm{CE}, \mathrm{AF}, \mathrm{PN}$ & C, S & - & BFG (2018) \\
\hline \multicolumn{6}{|l|}{ Neckeraceae Schimp. } \\
\hline Neckeropsis undulata (Hedw.) Reichardt & Neotropical & $\mathrm{AM}, \mathrm{CA}, \mathrm{CE}, \mathrm{AF}, \mathrm{PN}$ & $C, S$ & - & BFG (2018) \\
\hline \multicolumn{6}{|l|}{ Orthotrichaceae Arn. } \\
\hline${ }^{* *}$ Schlotheimia rugifolia (Hook.) Schwägr. & Neotropical & $\mathrm{AM}, \mathrm{CE}, \mathrm{AF}$ & C & Cerqueira 938 & - \\
\hline \multicolumn{6}{|l|}{ Pilotrichaceae Kindb. } \\
\hline${ }^{* *}$ Cyclodictyon varians (Sull.) Kuntze & Neotropical & $\mathrm{AM}, \mathrm{CE}, \mathrm{AF}$ & C & Sierra 4840 & - \\
\hline${ }^{* *}$ Hypnella pallescens (Hook.) A.Jaeger & Neotropical & $\mathrm{AM}, \mathrm{CE}, \mathrm{AF}$ & Ex & Sierra 10212 & - \\
\hline \multicolumn{6}{|l|}{ Phyllodrepaniaceae Crosby } \\
\hline${ }^{* *}$ Mniomalia viridis (Mitt.) Müll. Hal. & South-American & $\mathrm{AM}, \mathrm{CE}$ & C & Sierra 4829 & - \\
\hline \multicolumn{6}{|l|}{ Pottiaceae Schimp. } \\
\hline Trichostomum tenuirostre (Hook. \& Taylor) Lindb. & Neotropical & $\mathrm{AM}, \mathrm{CA}, \mathrm{CE}, \mathrm{AF}$ & C, $\mathrm{T}$ & - & BFG (2018) \\
\hline \multicolumn{6}{|l|}{ Pterobryaceae Kindb. } \\
\hline Henicodium geniculatum (Mitt.) W.R.Buck & Neotropical & $\mathrm{AM}, \mathrm{CE}, \mathrm{AF}, \mathrm{PN}$ & C & - & Lisboa (1976) \\
\hline Pireella pohlii (Schwägr.) Cardot & Neotropical & $\mathrm{AM}, \mathrm{CE}, \mathrm{AF}$ & C & - & BFG (2018) \\
\hline \multicolumn{6}{|l|}{ Pylaisiadelphaceae Goffinet \& W.R.Buck } \\
\hline Isopterygium tenerum (Sw.) Mitt. & Neotropical & $\mathrm{AM}, \mathrm{CA}, \mathrm{CE}, \mathrm{AF}, \mathrm{PA}, \mathrm{PN}$ & $C, S$ & - & BFG (2018) \\
\hline Taxithelium planum (Brid.) Mitt. & Afro-American & $\mathrm{AM}, \mathrm{CE}, \mathrm{AF}, \mathrm{PN}$ & $C$ & - & Sierra et al. (2018) \\
\hline \multicolumn{6}{|l|}{ Sematophyllaceae Broth. } \\
\hline $\begin{array}{c}\text { Brittonodoxa subpinnata (Brid.) W.R. Buck, } \\
\text { P.E.A.S.Câmara \& Carv.-Silva }\end{array}$ & Pantropical & $\mathrm{AM}, \mathrm{CA}, \mathrm{CE}, \mathrm{AF}, \mathrm{PA}, \mathrm{PN}$ & $C, E x, S$ & - & Griffin (1979) \\
\hline Meiothecium revolubile Mitt. & South-American & $\mathrm{AM}, \mathrm{CE}, \mathrm{AF}$ & C & - & Lisboa (1976) \\
\hline Microcalpe subsimplex (Hedw.) W.R.Buck & Neotropical & $\mathrm{AM}, \mathrm{AF}, \mathrm{CA}, \mathrm{CE}, \mathrm{PN}$ & $C, E x, T$ & Cerqueira 881 & $\begin{array}{l}\text { Lisboa (1976) } \\
\text { Griffin (1979) }\end{array}$ \\
\hline Trichosteleum. papillosum (Hornsch.) A.Jaeger & South-American & $\mathrm{AM}, \mathrm{CE}, \mathrm{AF}$ & C & Cerqueira 1211 & Griffin (1979) \\
\hline${ }^{* *}$ T. subdemissum (Besch.) A.Jaeger & South-American & $\mathrm{AM}, \mathrm{CE}, \mathrm{AF}$ & $C, S$ & Sierra 4857 & - \\
\hline T. vicentinum (Mitt.) A.Jaeger & Neotropical & $\mathrm{AM}$ & $C$ & - & Sierra et al. (2018) \\
\hline \multicolumn{6}{|l|}{ Sphagnaceae Dumort. } \\
\hline Sphagnum atroligneum H.A.Crum & Endemic to Brazil & $\mathrm{AM}, \mathrm{AF}$ & $S, T$ & - & BFG (2018) \\
\hline
\end{tabular}




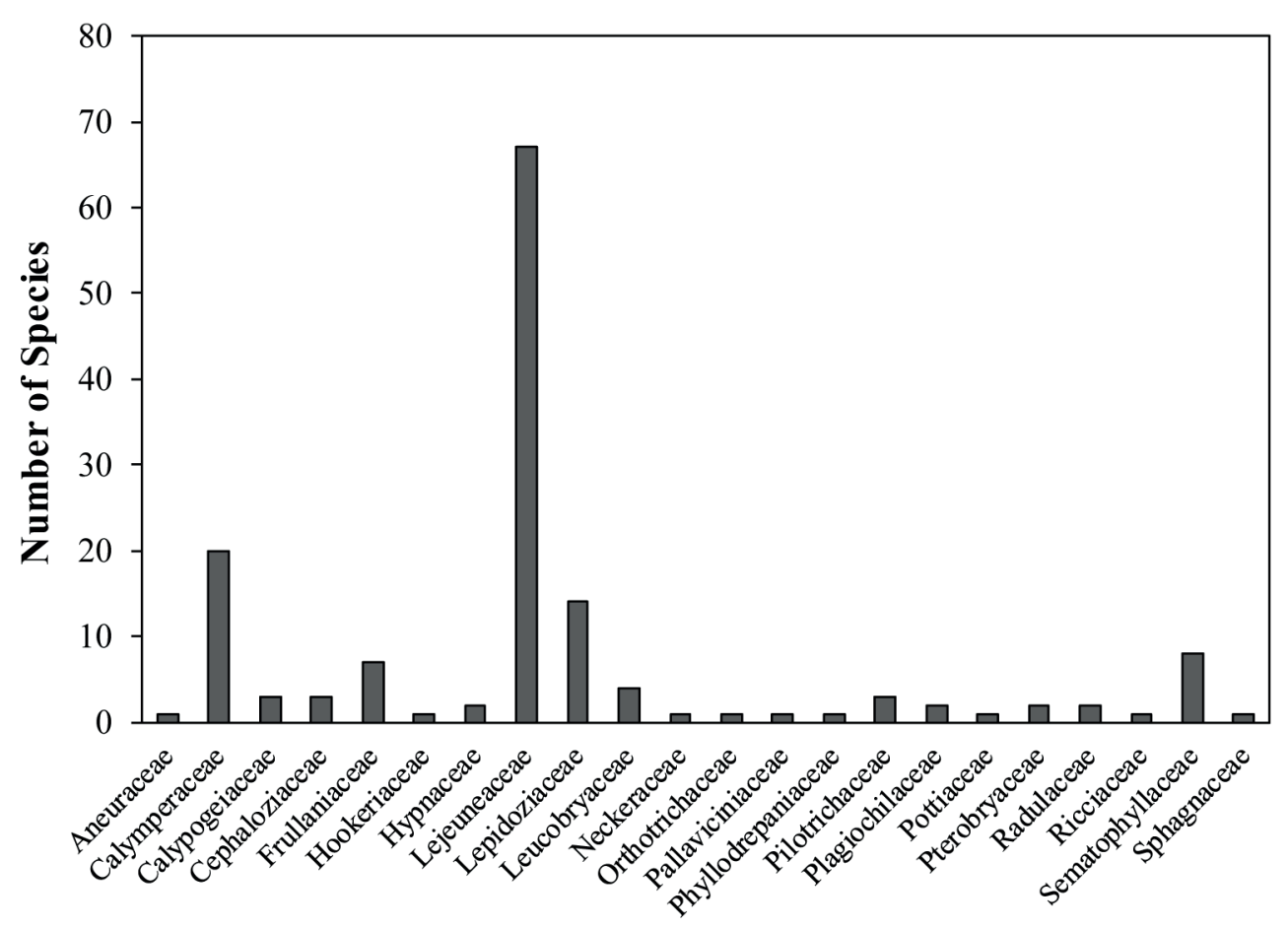

Figure 4. Species richness of bryophyte families in Amazonian Campinaranas.

this substrate in tropical forests (Richards 1984). Favored by high humidity of tropical environments, this substrate provides ideal microclimatic conditions for the development of bryophytes (Frahm 2003).

The bryophytes listed in this study present predominantly Neotropical distribution pattern (70 spp.), but also South American (13 spp.), Pantropical (15 spp.), Amazonian (14 spp.), Endemic to Brazil (03 spp.), Afroamerican (11 spp.), distribution in Amazonian-Guyanas (6 spp.), Tropical South American (5 spp.), Amazonian (+ Bahia) (2 spp.), and Amazonian (+ Costa Rica), S Europe, SW Asia and Trinidad (one species each).

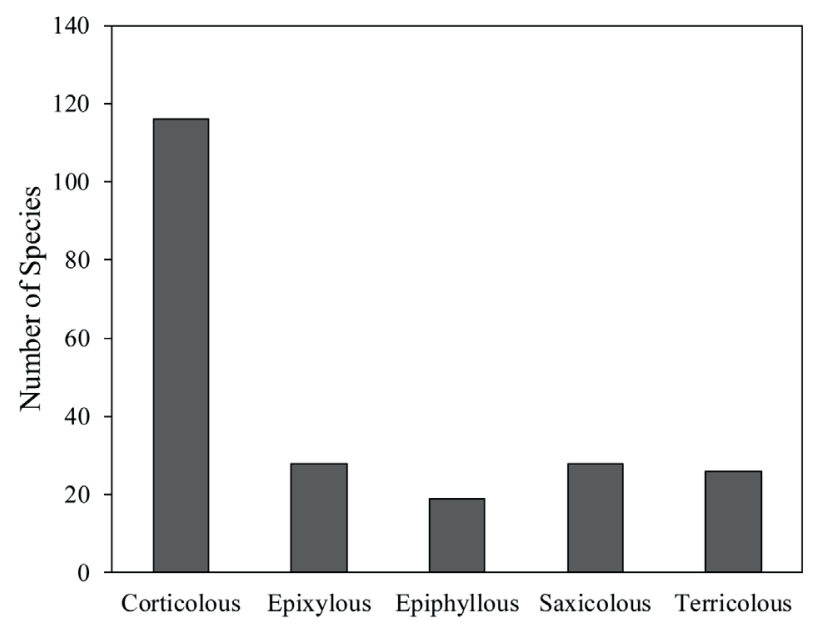

Figure 5. Distribution of species according to the substrates that they were collected in Amazonian Campinaranas.
Twenty-nine species are in Brazil exclusive to the Amazonian domain. Among them, the species Calypogeia tenax, Mnioloma caespitosa, Archilejeunea ludoviciana, Cheilolejeunea asperifolia, C. fragrantíssima, C. neblinensis, C. papulosa, Diplasiolejeunea buckii, Drepanolejeunea polyrhiza, Pycnolejeunea remotistipula, Verdoornianthus marsupiifolius, Micropterygium parvistipulum, Pteropsiella frondiformis, Telaranea pecten, Calymperes bartramii, Octoblepharum stramineum, Syrrhopodon annotinus, S. fimbriatus and $S$. flexifolius were thus far known from Terra Firme forest only, and had, therefore, their range of distribution extended to Campinarana vegetation with the present work.

This study emphasizes the importance of carrying out floristic inventories in poorly known environments and of further studies with different approaches, such as ecological, phytogeographic and genetic.

\section{Acknowledgements}

We thank to the PPG-Botânica and INPA for providing the infrastructure for species identification; the INPA/ MAUA Group, PELD (CNPq/CAPES/FAPS/BC, process 441590/2016-0), FAPEAM (PELD/FAPEAM, process 062.01357/2017), LBA Experiment in Amazonia, and the staff of the ATTO Project for financial and logistic support in the fieldwork; to the Fúvio Rubens Oliveira-da-Silva and S.R. Gradstein for critically reading the manuscript. This study was financed in part by the Coordenação de Aperfeiçoamento de Pessoal de Nível Superior - Brasil (CAPES) Finance Code 001. 


\section{Gabriela Ramos Cerqueira, Adriel M. Sierra, Anna Luiza Ilkiu-Borges, Sylvia Mota-de-Oliveira and Charles Eugene Zartman}

\section{References}

Adeney JM, Christensen NL, Vicentini A, Cohn-Haft M. 2016. White-sand ecosystems in Amazonia. Biotropica 48: 7-23.

Álvarez-Alonso J, Metz MR, Fine PVA. 2013. Habitat specialization by birds in Western Amazonian white-sand forests. Biotropica 45: 365-372.

Anderson AB, Prance GT, Albuquerque BWP. 1975. Estudos sobre a vegetação das campinas amazônicas III: a vegetação lenhosa da Campina da Reserva Biológica INPA SUFRAMA (Manaus Caracaraí, km 62). Acta Amazonica 5: 225-246.

Anderson AB. 1981. White-sand vegetation of Brazilian Amazonia. Biotropica 13: 199-210.

Barbé M, Chavel EE, Fenton NJ, et al. 2016. Dispersal of bryophytes and ferns is facilitated by small mammals in the boreal forest. Écoscience 23: 67-76.

Bastos CJP, Sierra AM, Zartman CE. 2016. Three new species of Cheilolejeunea (Spruce) Steph. (Marchantiophyta, Lejeuneaceae) from northern Brazil. Phytotaxa 277: 36-46.

Bastos CJP, Zartman CE. 2017. A new species of Pycnolejeunea (Marchantiophyta, Lejeuneaceae) from Brazil. Neodiversity 10: 1-6.

Bastos CJP, Gradstein SR. 2020. The genus Cheilolejeunea (Marchantiophyta: Lejeuneaceae) in tropical America. Nova Hedwigia 111: 287-335.

Bastos CJP. 2012. Taxonomia e distribuição de Cheilolejeunea aneogyna (Spruce) A. Evans (Lejeuneaceae, Marchnatiophyta). Acta Botanica Brasilica 26: 709-713.

Benavides JC, Duque A, Duivenvoorden JF, Cleef AM. 2006. Species richness and distribution of understory bryophytes in different forest types in Colombia Amazonia. Journal of Bryology 28: 182-189.

BFG - The Brazil Flora Group. 2018. Brazilian Flora 2020: Innovation and collaboration to meet Target 1 of the Global Strategy for Plant Conservation (GSPC). Rodriguésia 69: 1513-1527.

Braga PI. 1978. Estudos da flora orquidológica do Estado do Amazonas III - X Brassocattleya rubyi Braga (Orchidaceae) híbrido natural novo da flora amazônica. Acta Amazonica 8: 371-378.

Bramley-Alves J, King DH, Robinson SA. 2014. Dominating the Antarctic environment: Bryophytes in a time of change. In: Hanson DT, Rice SK. (eds.) Photosynthesis in Bryophytes and early land plants. Advances in Photosynthesis and Respiration. Dordrecht, Springer. p. 309-324.

Buck WR. 2003. Guide to the Plants of Central French Guiana - Part 3. Mosses. Memoirs of The New York Botanical Garden 76: 26-123.

Carmo DM, Peralta DF. 2016. Survey of bryophytes in Serra da Canastra National Park, Minas Gerais, Brazil. Acta Botanica Brasilica 30: 254-265.

Carvalho-Silva M, Stech M, Soares-Silva LH, et al. 2017. A molecular phylogeny of the Sematophyllaceae sl (Hypnales) based on plastid, mitochondrial and nuclear markers, and its taxonomic implications. Taxon 66: 811-831.

Coomes DA, Grubb PJ. 1996 Amazonian caatinga and related communities at La Esmeralda, Venezuela: forest structure, physiognomy and floristics, and control by soil factors. Vegetatio 122: 167-191.

Crandall-Stotler B, Stotler R, Long D. 2009. Morphology and classification of the Marchantiophyta. In: Goffinet B, Shaw AJ. Bryophyte Biology. Cambridge, Cambridge University Press. p. 1-54.

Dauphin G. 2003. Ceratolejeunea (Lejeuneaceae: Lejeuneoideae). Flora Neotropica Monograph 90: 1-86.

Ducke A, Black GA. 1954. Notas sobre a fitogeografia da Amazônia brasileira. Boletim Técnico Instituto Agronômico do Norte 29: 1-62.

Ducke A. 1922. Plantes Nouvelles ou Peu connues de la Région Amazonienne. Archivos do Jardim Botânico do Rio de Janeiro 3: 3-269.

Ellwanger JH, Kulmann-Leal B, Kaminski VL, et al. 2020. Beyond diversity loss and climate change: Impacts of Amazon deforestation on infectious diseases and public health. Annals of the Brazilian Academy of Sciences 92: 1-33.

Egler WA. 1960. Contribuição ao conhecimento dos campos da Amazônia. 1 - Os campos do Ariramba. Boletim do Museu Paraense Emílio Goeldi, Série Botânica 4: 1-36.

Frahm JP. 2003. Manual of tropical bryology. Tropical Bryology 23: 1-196.

Fulford MH. 1966. Manual of the leafy Hepaticae of Latin America. Memoirs of the New York Botanical Garden 11:173-276.
Garcia ET, Oliveira SM, Tavares-Martins ACC, Pôrto KC. 2020. Vertical gradient of epiphytic bryophytes in the Amazon: the rule and its exception. Cryptogamie, Bryologie 41: 55-65.

García-Villacorta R, Dexter KG, Pennington T. 2016. Amazonian whitesand forests show strong floristic links with surrounding oligotrophic habitats and the Guiana Shield. Biotropica 48: 47-57.

Goffinet B, Buck WR, Shaw JA. 2009. Morphology, anatomy, and classification of the Bryophyta. In: Goffinet B, Shaw AJ. (eds.) Bryophyte Biology. Cambridge, Cambridge University Press. p. 55-138.

Gradstein SR, Churchill SP, Salazar-Allen N. 2001. Guide to the bryophytes of tropical America. Memoirs of the New York Botanical Garden 86: 1-577.

Gradstein SR, Costa DP. 2003. The Hepaticae and Anthocerotae of Brazil. Memoirs of the New York Botanical Garden 88: 1-673.

Gradstein SR, Ilkiu-Borges AL. 2009. Guide to the plants of Central French Guiana. Part 4. Liverworts and hornworts. Memoirs of the New York Botanical Garden 76: 1-140.

Gradstein SR, Ilkiu-Borges AL. 2015. A taxonomic revision of the genus Odontoschisma (Marchantiophyta: Cephaloziaceae). Nova Hedwigia 100: $15-100$

Gradstein SR, Uribe J. 2011. A synopsis of the Frullaniaceae (Marchantiophyta) from Colombia. Caldasia 33: 367-396.

Gradstein SR. 1994. Lejeuneaceae: Ptychantheae, Brachiolejeuneae. Flora Neotropica Monograph 62: 1-216.

Gradstein SR. 2013. Afro-Amerian Hepatics revisited. Polish Botanical Journal 58: 149 -177.

Gradstein SR. 2016. The genus Plagiochila (Marchantiophyta) in Colombia. Revista de La Academia Colombiana de Ciencias Exactas, Físicas y Naturales 40: 104-136.

Gradstein SR. 2017. Bazzania (Marchantiophyta) in South America. Nova Hedwigia 105: 1-24.

Griffin D. 1975. The Bryology of a Brazilian campina forest. The Association of Southeastern Biologists Bulletin 22: 1-55.

Griffin D. 1979. Guia preliminar para as briófitas frequentes em Manaus e adjacências. Acta Amazonica 9: 1-67.

Guevara JE, Damasco G, Baraloto C, et al. 2016. Low phylogenetic beta diversity and geographic neo-endemism in Amazonian white-sand forests. Biotropica 48: 34-46.

He XL. 1999. A taxonomic monograph of the genus Pycnolejeunea (Lejeuneaceae, Hepaticae). Acta Botanica Fennica 163: 1-77.

IBGE - Instituto Brasileiro de Geografia e Estatística. 2012. Manual técnico da vegetação brasileira. 2nd. edn. Rio de Janeiro, Instituto Brasileiro de Geografia e Estatística - IBGE.

Ilkiu-Borges AL .2006. A taxonomic monograph of the genus Prionolejeunea (Lejeuneaceae Jungermanniopsida). Göttingen, Cuvillier Verlag.

Ilkiu-Borges AL, Lisboa RCL. 2004. Os gêneros Cyclolejeunea, Haplolejeunea, Harpalejeunea, Lepidolejeunea e Rectolejeunea (Lejeuneaceae, Hepaticae) na Estação Científica Ferreira Penna, Pará, Brasil. Acta Botanica Brasilica 18: 539-555.

Ireland RR, Buck WR. 1994. Stereophyllaceae. Flora Neotropica Monograph 65: 1-49.

Klein VP, Piedade MTF. 2019. Orchidaceae occurring in white-sand ecosystems of the Uatumã Sustainable Development Reserve in Central Amazon. Phytotaxa 419: 113-148.

Koury CG, Rizzo E, Albuja MG. 2012. O turismo na Reserva de Desenvolvimento Sustentável do Uatumã: conjuntura atual e possibilidades de geração de renda para as comunidades locais. Manaus, Conservação e Desenvolvimento Sustentável - IDESAM.

Lima E, Oliveira-da-Silva FR, Ilkiu-Borges AL. 2018. Flora das cangas da Serra dos Carajás, Pará, Brasil: Frullaniaceae. Rodriguésia 69: 973-981.

Lisboa RCL. 1976. Estudos sobre a vegetação das campinas amazônicas V: Brioecologia de uma campina amazônica. Acta Amazonica 6: 171-191.

Lönnel N, Hylander K, Jonsson BG, Sundberg S. 2012. The fate of the missing spores-patterns of realized dispersal beyond the closest vicinity of a sporulating moss. PLOS ONE 7: e41987. doi: 10.1371/ journal.pone.0041987.

Mota-de-Oliveira S, ter Steege H. 2015. Bryophyte communities in the Amazon forest are regulated by height on the host tree and site elevation. Journal of Ecology, 103: 441-450. 


\section{Unveiled diversity: Amazonian Campinaranas harbor twice the number of bryophyte species recorded in the last century}

Oliveira-da-Silva FR, Ilkiu-Borges AL. 2018. Briófitas (Bryophyta e Marchantiophyta) das cangas da Serra dos Carajás, Pará, Brasil. Rodriguésia 69: 1405-1416.

Pereira MRS. 2019. Avanços florísticos e filogênicos de Calymperaceae Kindb. (Bryophyta) para Amazônia. PhD Thesis, Instituto Nacional de Pesquisas da Amazônia, Manaus.

Pires JM, Prance GT. 1985. The vegetation types of the Brazilian Amazon. In: Prance GT, Lovejoy TE. (eds.) Key Environments: Amazonia. 1st. edn. Oxford, Pergamon Press. p. 109-145.

Pócs T, Bernecker A, Tixier P. 2014. Synopsis and key to species of Neotropical Cololeijeunea (Lejeuneaceae). Acta Botanica Hungarica 56: 185-226.

Pócs T. 2016. Contribution to the bryoflora of Australia. VI. The genus Cololejeunea (Spruce) Steph. (Lejeuneaceae, Marchantiophyta). Polish Botanical Journal 61: 205-229.

Prance GT. 1996. Islands in Amazonia. Philosophical Transactions of the Royal Society of London, series B 351: 823-833.

Reese WD. 1993. Calymperaceae. Flora Neotropica Monograph 58: 1-102.

Reichardt K, Santos A, Nascimento-Filho V, Bacc OOS. 1975. Movimento de água subterrânea em ecossistema campina amazônica. Acta Amazonica 6: 229-290.

Richards WP. 1984. The ecology of tropical forest bryophytes. In: Schuster RM. (ed.) New Manual of Bryology. Japan, Hattori Botanical Laboratory. p. 1233-1270.

Robbins RG. 1952. Bryophyta Ecology of a dune area in New Zealand vegetation. Acta Geobotânica 4: 1-131.

Santos ND, Costa DP, Kinoshita LS, Shepherd GJ. 2011. Aspectos brioflorísticos e fitogeográficos de duas formações costeiras de Floresta Atlântica da Serra do Mar, Ubatuba/ SP, Brasil. Biota Neotropica 11: 425-438.
Santos ND, Costa DP. 2010. Phytogeography of the liverwort flora of the Atlantic Forest of southeastern Brazil. Journal of Bryology 32: 9-22.

Shi XQ, Zhu RL. 2015. A revision of Archilejeunea s.str. (Lejeuneaceae, Marchantiophyta). Nova Hedwigia 100: 589-601.

Shi XQ, Gradstein SR, Zhu RL. 2015. Type studies on Archilejeunea (Lejeuneaceae, Marchantiophyta): Five new synonyms and a new combination. Phytotaxa 195: 248-250. doi: 10.11646/phytotaxa.195.3.5.

Sierra AM, Vanderpoorten A, Gradstein SR, Pereira MR, Bastos CJP, Zartman CE. 2018. Bryophytes of Jaú National Park (Amazonas, Brazil): Estimating species detectability and richness in a lowland Amazonian megareserve. The Bryologist 121: 571-588.

Silveira M. 2017. O complexo vegetacional sobre areia branca no Alto Juruá. In: Brito TF, Silva RC, Oliveira SAV, Silveira M. (eds.) Complexo vegetacional sobre areia branca: Campinaranas do Sudoeste da Amazônia. Rio Branco, Editora da Universidade Federal do Acre - Edufac. p. 11-19.

Smith RJ, Stark LR. 2014. Habitat vs. dispersal constraints on bryophyte diversity in the Mojave Desert, USA. Journal of Arid Environments 102: $76-81$.

Spruce R. 1884. Hepaticae Amazonicae et Andiane. Transactions and Proceedings of Society of Edinburgh 15: 1-308.

Vittoz P, Camenisch M, Mayor R, Miserere L, Vust M, Theurillat JP. 2010. Subalpine-nival gradient of species richness for vascular plants, bryophytes and lichens in the Swiss Inner Alps. Botanica Helvetica 120: 139-149.

Yano O. 1984. Briófitas. In: Fidalgo O, Bononi VLR. (eds.) Técnicas de coleta, preservação e herborização de material botânico. São Paulo, Instituto de Botânica.

Yano O. 1992. Leucobryaceae (Bryopsida) do Brasil. PhD Thesis, Universidade de São Paulo, São Paulo. 